\title{
Investigation of the temperature behaviour of sliding rubber materials
}

\author{
O. Lahayne \& J. Eberhardsteiner \\ Vienna University of Technology, \\ Institute for Mechanics of Materials and Structures (IMWS), \\ Vienna, Austria
}

\begin{abstract}
At the IMWS a testing device called Linear Friction Tester (LFT) was developed, which is mainly used to characterise and quantify the friction behaviour of tire materials under varying conditions. During the friction process a considerable amount of heat is generated at the contact surface. Starting from theoretical approaches, there is supposed to be a strong interrelation between the friction coefficient $\mu$ and the temperature $T$ of the rubber. By means of temperature sensors, FE-calculations and a model for the heat conduction it was possible to measure and calculate the heating of the rubber with high accuracy. In this paper some examples are presented for results of such measurements. A model for the heat conduction is illustrated, and testing the correlation between measurement and calculations validates the practicability of the model.

Keywords: Rubber, Temperature, Friction, Tire.
\end{abstract}

\section{The testing device}

In 1997 the Linear Friction Tester has been constructed [3]. The test conditions at the LFT are well defined, since it is placed in an air-conditioned container, and in good agreement with realistic breaking situations. The core of the device is a linear drive that pulls a sledge with a rubber test specimen over the friction surface. A pneumatic system applies the vertical load (up to $2000 \mathrm{~N}$ or 10 bar). The test specimens have a size between 10 and $30 \mathrm{~cm}^{2}$ and contain different arrangements of lamellae. The friction surfaces were cut out of real road surfaces. Also friction tests on snow and ice surfaces can be performed. For some experiments additionally a high-speed-camera recorded the deformation of the sample. 


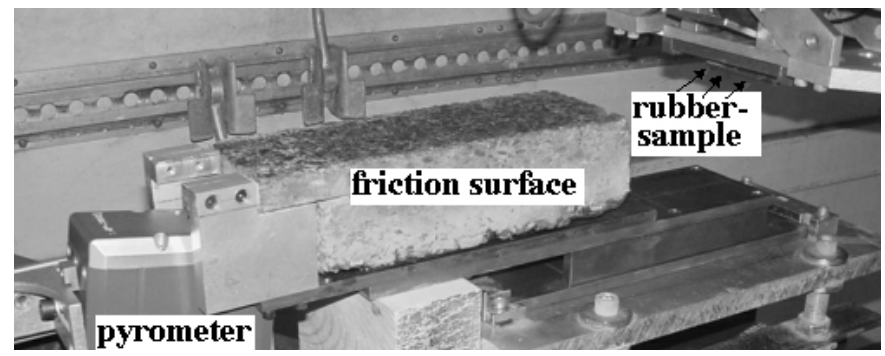

Figure 1: $\quad$ Friction surface with pyrometer and rubber sample.

The temperature of the rubber samples was recorded in two ways: If the temperature at the friction surface was of interest, an infrared-Pyrometer was used. It has a resolution of $1,4 \mathrm{~mm}$ and a response time of $5 \mu \mathrm{s}$, which is adequate for the size and sliding velocities of the rubber samples.

The temperature within the samples was recorded with up to four thermocouples, which were implanted in the samples. In both cases the signals of the measurement device were synchronised with the position of the samples.

\section{Theory}

Basis for the analytical calculation of the heat conduction within the samples is Fourier's law of heat conduction:

$$
\dot{T}(x, t)=\frac{\lambda_{m}}{c_{m} \rho_{m}} T^{\prime \prime}(x, t)=a_{m} T^{\prime \prime}(x, t)
$$

In this one-dimensional version, $T$ denotes the temperature, $\lambda$ the heat conductivity, $c$ the heat capacity, $\rho$ the density, and $a$ the temperature conductivity. $x$ is the distance to the friction surface, as shown in Figure 2. If this one-dimensional version is oversimplifying the problem will be discussed later. The maximum temperature within the sample is called $T_{R}$; the environment temperature $T_{0}$. Based on the assumption that the carrier material will always have the temperature $T_{0}$, the stable end condition after a sufficient friction time is a linear decrease of the temperature within the sample between $T_{R}$ and $T_{0}$, as plotted in Figure 2. $v_{\text {slide }}$ is the sliding velocity, $F_{x}$ the vertical load.

As a first step, the process of heat conduction was calculated by means of a heat-flow-simulation based on Fourier's equation. For a one-dimensional simulation, the distance between the friction surface and the carrier material was divided in intervals of the same length; for two dimensions the cross-section was divided into squares. For the example in Figure 3, the absorbed power was highest in the middle of the sample and zero at the corners. 


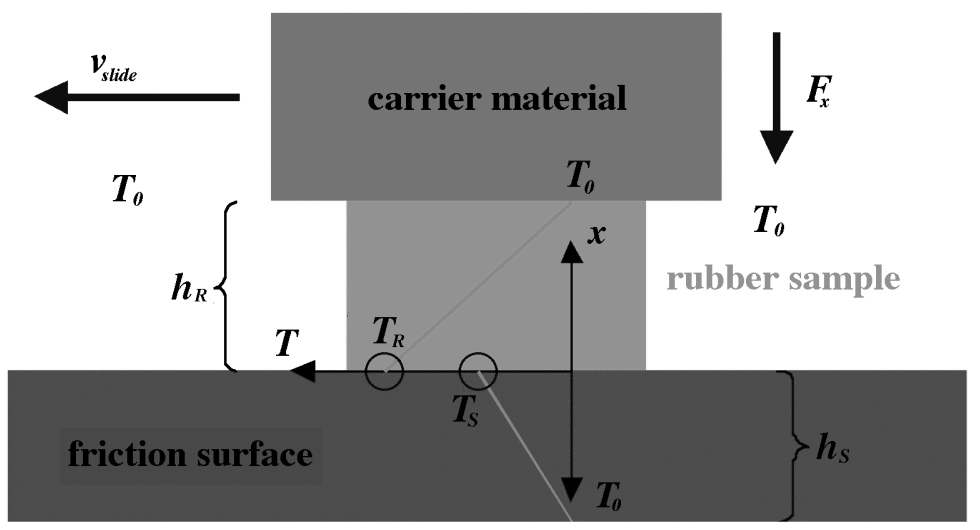

Figure 2: $\quad$ Schema of heat conduction during friction process.

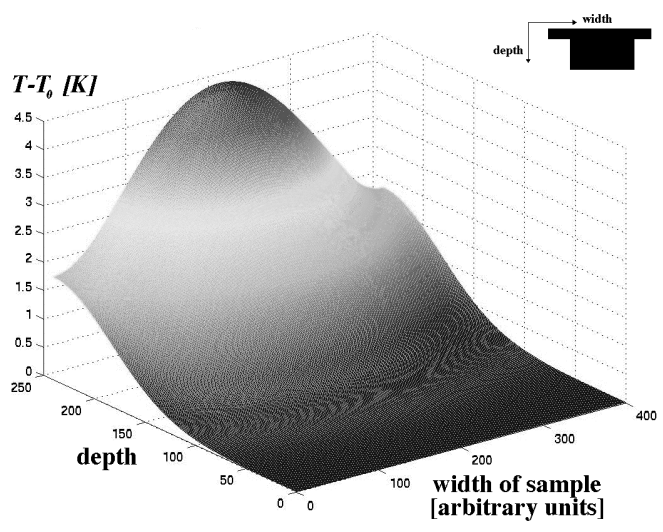

Figure 3: Result of simulation of heat conduction.

The results of the measurements and the heat-flow simulation were compared to each other and to an analytical solution of Fourier's equation. The boundary conditions for all methods were the same. For the analytical solution you have to distinguish between the process of heating and the process of cooling. Equation (2) shows the exact solution for Equation (1) for the proper boundary conditions.

$$
\begin{aligned}
& T_{W}(x, t)=T_{0}-U_{m}\left(1-\frac{x}{h_{G}}\right)+\frac{8}{\pi^{2}} U_{m} \sum_{k=0}^{\infty} \frac{1}{(2 k+1)^{2}} \cos \left(n_{k} x\right) \cdot e^{-n_{k}{ }^{2} a t} \\
& T_{A}(x, t)=T_{0}+\frac{8}{\pi^{2}} U_{m} \sum_{k=0}^{\infty}\left[\frac{1}{(2 k+1)^{2}}-\frac{1}{\pi} \sum_{l=0}^{\infty} \frac{1}{(2 l+1)^{2}} \cdot e^{-n_{l}{ }^{2} a t_{r}}\left(\frac{1}{l+k}+\frac{1}{l-k}\right)\right] \cos \left(n_{k} x\right) \cdot e^{-n_{k}{ }^{2} a t} \\
& n_{k}=\frac{2 k+1}{h_{G}} \frac{\pi}{2}, U_{m}=\frac{P_{R} h_{R}}{\lambda a}
\end{aligned}
$$


$P_{R}$ is the power generated by the friction process and absorbed by the rubber. The first line of Equation (2) is the solution for the process of heating; the second line the solution for the process of cooling.

Since these solutions are a bit unwieldy, for some tasks a crude approximation for the process of heating has been used:

$$
T_{W}(x, t)=\frac{P_{R}}{\lambda A} \sqrt{2 a t} \cdot e^{-\frac{x}{\sqrt{2 a t}}}+T_{0} .
$$

This approximation is usable only for $x<<\sqrt{2 a t}$.

The power generated by the friction process is given by

$$
P_{\text {tot }}=F_{y} \cdot \frac{s}{t}=F_{y} \cdot v_{\text {Slide }}=\mu \cdot F_{x} \cdot v_{\text {Slide }} .
$$

Most of the generated power is absorbed by the friction surface; the rubber absorbs only about $1 \%[4,5]$. Because the partition of $P_{t o t}$ between rubber and surface wasn't precisely known, it was for some tasks fitted to the results. Alternatively $P_{R}$ can be calculated by means of FE- calculations, as Figure 5 will show.

\section{Results}

\subsection{Temperature within the samples}

It was helpful to test the correctness of the solution of Fourier's equation first not during a friction process, but by heating up a rubber sample at one side. In this way it is possible to reach higher temperatures without the vibrations of a friction process. For this, a small heating element was used, which affected only the surface of the sample, while the opposite surface was fixed on the carrier material. The temperature data were measured by four thermocouples. Figure 4 shows the sample and the position of the sensors.

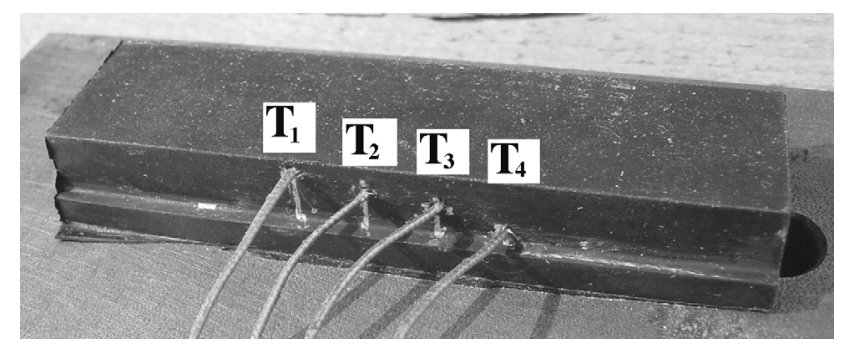

Figure 4: Rubber sample with thermocouples. 
Figure 5 shows the results of a test with this sample. The applied temperature was $55 \mathrm{~K}$ above $T_{0}$.

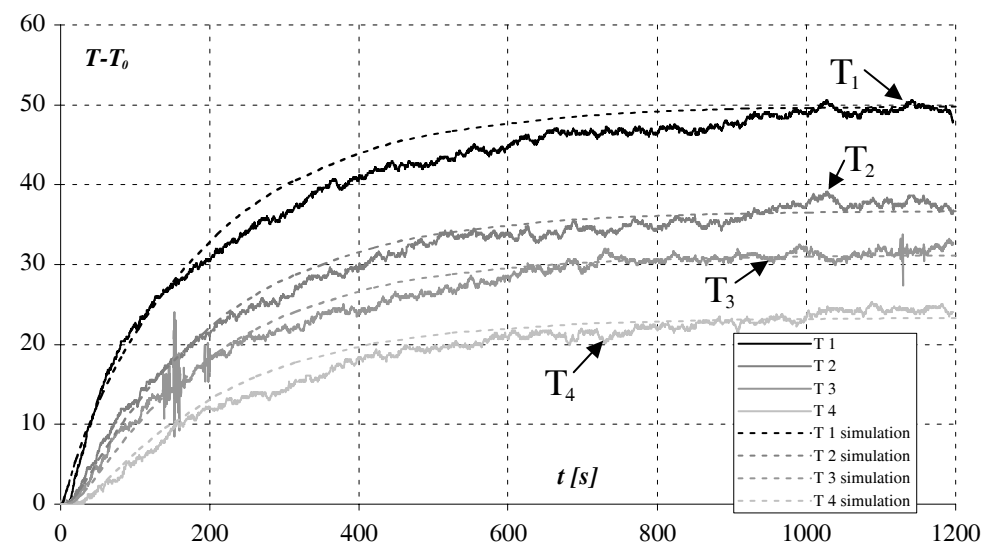

Figure 5: Temperature within the sample during heating.

The solid lines show the measured temperature, the dashes lines the results of the solution based on Equation (2) belonging to the position of the thermocouples T1-T4. The fit between measurement and calculation is satisfactory.

For this test the surface has been heated up homogeneous. For a friction process, though, an inhomogeneous distribution of the temperature at the surface has to be expected because of the deformation of the sample. Since the periods of heating up are much shorter during friction processes, the rise of the temperature is much smaller than in Figure 5, as Figure 6 demonstrates.

Figure 6 shows the results for two measuring points at distances of $0.3 \mathrm{~mm}$ (T3) and $0.7 \mathrm{~mm}$ (T1) from the surface. The data are compared with

- the results of a one-dimensional heat-flow-simulation (1D),

- the results of a two-dimensional simulation (2D), as shown in Figure 3,

- the analytical solution of Fourier's equation as shown in Equation (2).

As it can be seen, the results of the three methods are equivalent within the limits of the statistical spread of the measured data.

The length of the friction surface, as shown in Figure 1, was limited to 300 $\mathrm{mm}$. Because of that only comparatively low temperatures were measured within the sample, especially if the distance of the sensor to the friction surface was higher than $1 \mathrm{~mm}$. At the surface of the samples itself the temperature was always maximal. Because of that more precise tests were possible by the help of the pyrometer, which measured the temperature at the surface of the samples. This, though, was only possible after the end of the friction process because of the installation of the pyrometer. 


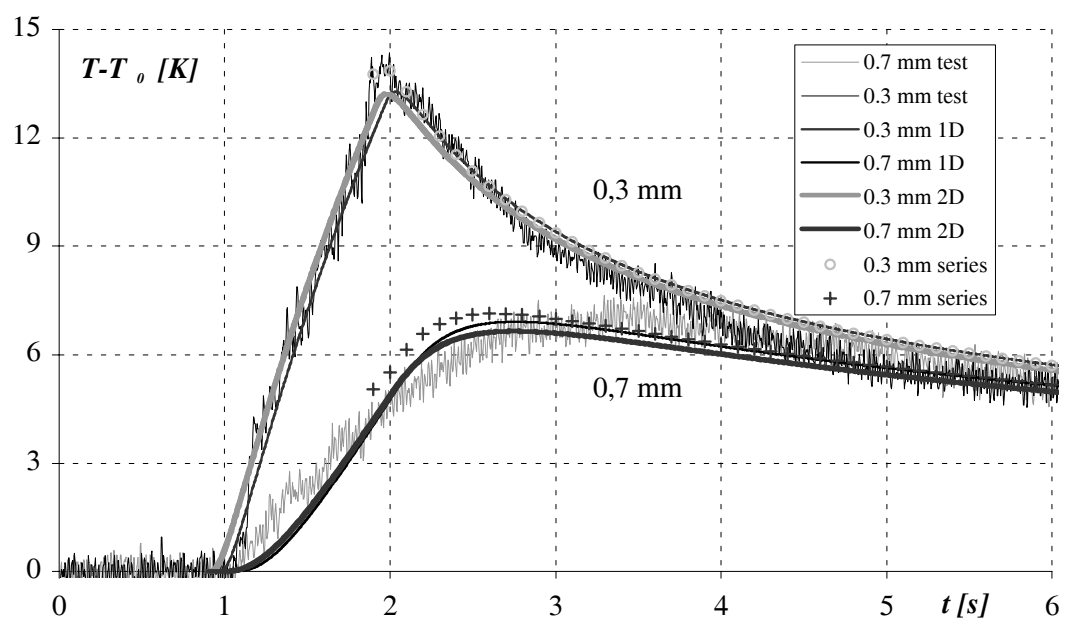

Figure 6: Temperature within the sample during friction process.

\subsection{Temperature at the friction surface}

The highest rises in temperature were measured for the roughest surfaces, such as asphalt. On this surface first a series of tests has been done to analyse the influence of the test parameters on the temperature. Not surprisingly, there is a strong influence of the vertical force, as can be seen in Figure 7. The vertical force was varied between 270 and $560 \mathrm{~N}$, resulting in pressures between 1.7 and 3.5 bar. Only for $420 \mathrm{~N}$ the measuring points were marked with crosses. On the abscissa the sliding distance was plotted. The width of the sample was $20 \mathrm{~mm}$.

In the same way the influence of other parameters was analysed, such as the influence of the sliding velocity, the sliding distance as well as the compound and the geometry of the sample. This demonstrates that the assumption for the heat distribution in Figure 3 is quite realistic.

These measurements were compared to results of FE-simulations of the temperature distribution, based on a material and friction model $[5,6]$. Crucial for the characteristics of $T(s)$ is the deformation behaviour of the rubber sample. Figure 8 shows the temperature along the centre-line of the sample, based on FE-simulations and on measurements for the same parameters. Except for the maximum at the leading edge (left, $-10 \mathrm{~mm}$ ) which can't be resolved by the sensor, there is a good correlation between measurement and calculation.

This example alludes to tests on a very rough surface, which causes high deformations of the rubber sample, especially at the leading edge. For this reason there is usually a maximum for $T(s)$ at the leading edge (at about $-12 \mathrm{~mm}$ ) and a higher maximum near the middle of the sample.

For the result in Figure 7, the rubber sample has been moved over the pyrometer; in this way the spatial distribution of the temperature was measured. For measuring the temporal run of the temperature, the sample was usually 
stopped near the maximum of $T(s)$, i.e. at about $135 \mathrm{~mm}$ for the examples in Figure 7. Figure 9 shows two examples for $T(t)$ for two sliding velocities, 300 and $700 \mathrm{~mm} / \mathrm{s}$.

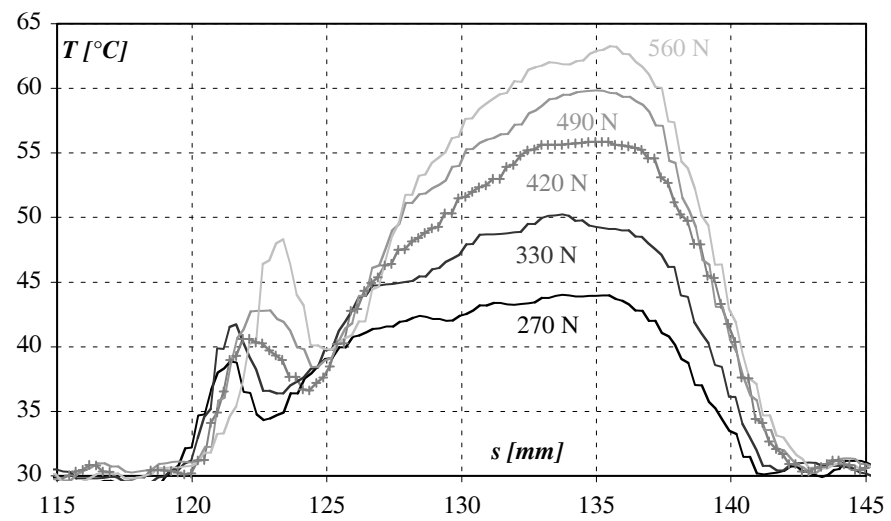

Figure 7: Influence of vertical force on the temperature.

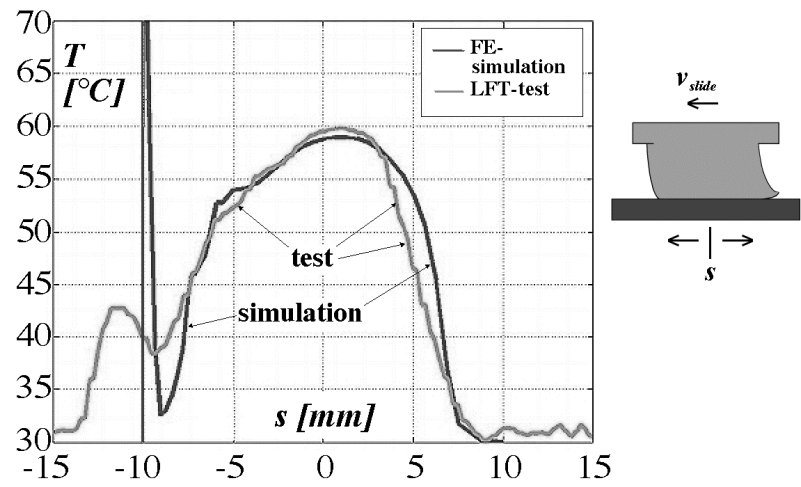

Figure 8: Comparison of FE- simulation and measurement.

The data of the measurements (solid lines) were compared to the results of the simulations, based on the parameters for the respective tests (circles). The results show that it is possible to describe and predict the temperature behaviour of a sliding rubber sample with satisfying accuracy, if the relevant parameters of the involved materials are given.

For some applications it is also possible to make good use of the approximation in Equation (3). In Figure 10, the mean value for three measurements for $T(t)$ as in Figure 9 has been calculated (black circles), together with the standard deviation (grey bars). Using $t^{0,5}$ as $\mathrm{x}$-axes, the diagram should show a linear run according to Equation (3). 


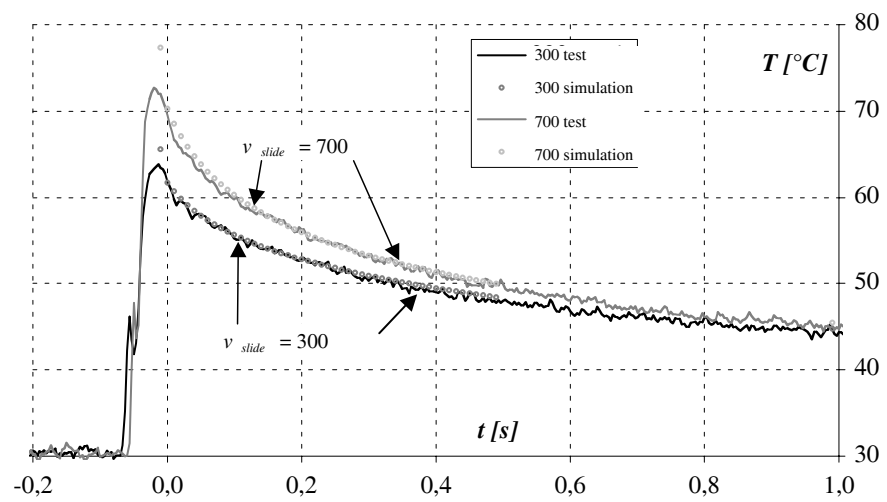

Figure 9: $\quad$ Examples for $T(t)$ at the surface.

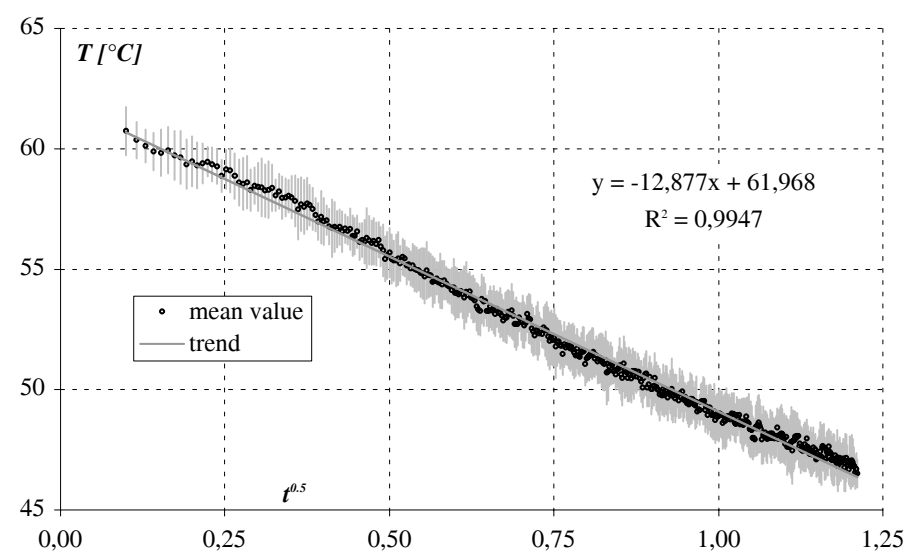

Figure 10: Example for approximation.

As can be seen, there is a good correlation between the straight line and the values for $T(t)$. Using this kind of analysis, the values of $a$ and $\lambda$ can be found.

These examples demonstrate the following:

- that the Equations (2) and (3) are correct solutions of Fourier's equation for heat conduction for these applications, and

- that the simulation of the heat conduction based on Equation (1) is equivalent to the exact solution in Equation (2).

Based on the results of the tests it is to be assumed that there is an inhomogeneous heat distribution over all cross-sections of the sample. In this case a higher number of thermocouples would be necessary to calculate the total flow of heat through the sample. Based on these results, it would be also possible to calculate the partition of the heat generated according to Equation (4) between surface and sample, which isn't known precisely so far. This affects only the total heat flow, and not the dependence of time and position within the sample. 


\section{Prospects}

As mentioned before, the main focus of the tests at the LFT was the determination of the friction coefficient of tire materials. But tests at the LFT as well as theoretical studies have shown that the temperature of the sample has a great influence on the friction coefficient, and so also on the road performance of tires. For that reason it is also important to determine the heat generated during friction processes. In fact also for tires a high rise in temperature was measured, especially for breaking processes $[7,8]$. Since it is easier to measure the temperature at the LFT these tests are helpful to improve the predictability of the road performance of tires.

The measurement of the temperature at the sample surface with a pyrometer is precise enough for our demands, but the tests with thermocouples can still be improved to gather more information about the three-dimensional heat flow within the samples during and after the friction processes. So far also a satisfactory description of the partition of the generated heat between sample and friction surface has to been found.

\section{Acknowledgements}

Most measurements for the mentioned experiments have been done with materials, which were produced and made available by the Continental AG, Hannover. This works wouldn't have been possible without the support of the colleagues of this company, especially Joachim Schramm, Bernhard Zernetsch and Reinhard Mundl. Therefore we want to express our gratitude for their support and cooperation and for their approval to make use of some of the results for this work.

\section{References}

[1] Olaf Lahayne: Experimentelle Reibungsuntersuchungen und Modellrechnungen zum Verhalten von Reifenmaterialien, $\mathrm{PhD}$ thesis, TU Wien 2007 (to be published)

[2] B.N.J. Persson: Role of the Flash Temperature; to be published 2007

[3] Harald Schwaiger: Entwicklung einer Prüfeinrichtung zur Untersuchung des Traktionsverhaltens von Gummiproben auf verschiedenen Oberflächen; Master thesis, TU Wien, 1996

[4] M. ten Bosch: Wärmeübertragung; Springer Verlag Berlin 1936

[5] K. Hofstetter: Thermo-mechanical Simulation of Rubber Tread Blocks during frictional Sliding, $\mathrm{PhD}$ thesis, TU Wien 2004

[6] K. Hofstetter, J. Eberhardsteiner, H.A. Mang, S. Del Linz: A ThermoMechanical Formulation Describing the Frictional Behavior of Rubber"; in: "Proceedings of the Fifth World Congress on Computational Mechanics (WCCM V) (Online-Proceedings http://wccm.tuwien.ac.at), H.A. Mang, F.G. Rammerstorfer, J. Eberhardsteiner (ed.); TU Wien, 2002 
164 Computer Methods and Experimental Measurements VIII

[7] Horst W. Stumpf: Reifenkonstruktion und Reifentechnik, lecture notes TU Wien, 2003

[8] N. Hagn: Messung der Temperaturverteilung im Latsch eines blockierten Reifens mittels Unterflurthermografie; TU Wien 1988/89 